\title{
ASCITIS QUILOSA ASOCIADA A PANCREATITIS AGUDA*
}

\author{
Drs. Beatriz Pérez C. ${ }^{1}$, Antonio Palomeque J. ${ }^{1}$, María Dolores Hernández G. ${ }^{1}$, \\ Francisco Navarro F. ${ }^{1}$, José Antonio Jiménez R. ${ }^{1}$ \\ 1 Unidad de Cirugía Hepatobiliopancreática. Servicio de Cirugía General y del Aparato Digestivo. \\ Hospital Universitario San Cecilio. \\ Granada, España.
}

\section{Chylous ascites associated with acute pancreatitis}

Background: Chylous ascites is defined as the presence of thoracic or intestinal lymph in the abdominal cavity. Its association with acute pancreatitis is uncommon. Aim: To report three cases of chilous ascites related with acute pancreatitis. Material and Methods: Review of medical records of three patients with chylous ascites associated with acute pancreatitis. Results: We report three patients with chylous ascites out of 2,188 admissions for acute pancreatitis $(0.13 \%)$. A 39 years old male with a pancreatic pseudocyst. During surgery, chylous ascites was found. He was successfully treated with octreotide and spironolactone. A 71 years male operated for an acute cholecystitis and a perivesicular abscess. Three days after surgery, a chylous fluid appears in the abdominal drainage, which disappears spontaneously. A 73 years old female operated for an acute pancreatitis. During surgery a chylous ascites is found. The patient died four days after surgery. Conclusions: Chylous ascites associated with pancreatitis is uncommon and octreotide may have a therapeutic role for it.

Key words: Pancreatitis, ascites, chylous.

\section{Resumen}

Introducción: La ascitis quilosa (AQ) se define como la presencia de linfa de origen torácico o intestinal en la cavidad abdominal. Asociada a Pancreatitis Aguda (PA), sólo se han documentado 12 casos hasta 2013. El objetivo de este trabajo es presentar tres nuevos casos y realizar una revisión de la presentación, clínica, diagnóstico y tratamiento de esta entidad. Material y Métodos: Se recogen los datos clínicos y evolutivos de tres pacientes con AQ secundaria a pancreatitis aguda (AQPA); esta se ha definido como la presencia de linfa en cavidad abdominal en un paciente con episodio de pancreatitis aguda reciente y sin antecedente traumático, infeccioso, neoplásico o quirúrgico que pudiera ser el responsable de la misma. Se han recogido igualmente todos los casos documentados hasta 2013. Resultados: En los últimos 10 años han sido ingresados 2.188 pacientes por PA entre los cuales, se han diagnosticado 3 casos de AQPA $(0,13 \%)$, dos hombres y una mujer, con PA de origen biliar. El diagnóstico ha sido "de visu" intra o postoperatorio. El débito inicial fue de

*Recibido el 27 de noviembre de 2011 y aceptado para publicación el 3 de marzo de 2014 .

Los autores declaran no tener conflicto de interés.

Correspondencia: Dra. Beatriz Pérez C.

bperezcabrera@ugr.es 
$3.000,300$ y $1.500 \mathrm{cc}$. El tratamiento ha contado con octeótrido de entrada en todos los casos. En la revisión bibliográfica la AQPA ha sido diagnosticada también "de visu" y los pacientes se han resuelto con tratamiento conservador, tardando una media de 27,7 días si se utiliza octeótrido frente a 46,5 días si no se le ha prescrito. Conclusiones: La AQ asociada a PA sigue siendo infrecuente, su diagnóstico es "de visu" y se resuelve con tratamiento conservador, siendo el octeótrido un fármaco de primera línea.

Palabras clave: Ascitis quilosa, quiloperitoneo, pancreatitis aguda, octeótrido.

\section{Introducción}

La ascitis quilosa (AQ), quilascos o quiloperitoneo se define como la presencia de linfa de origen torácico o intestinal en la cavidad abdominal ${ }^{1}$. Se produce como consecuencia de la obstrucción linfática con dilatación y extravasación de la linfa o por disrupción traumática de los vasos linfáticos; el diagnóstico se sospecha por la presencia de un líquido de aspecto lechoso y se confirma con el análisis bioquímico que detecta más de $120 \mathrm{mg} / \mathrm{dl}$ de triglicéridos. Su incidencia real es desconocida, entre $1: 20.000$ y $1: 180.000$ admisiones hospitalarias ${ }^{1,2}$ y la causa principal en el adulto es el traumatismo directo o quirúrgico de los vasos linfáticos; dentro de las causas no traumáticas, las más frecuentes son las neoplasias y la cirrosis ${ }^{1-3}$. Hasta hoy, solamente se han documentado 12 casos de ascitis quilosa asociada a pancreatitis aguda (AQPA): 5 de ellos a pancreatitis alcohólica ${ }^{4-8}, 2$ asociados a pancreatitis por hiperlipidemia, uno de ellos en una mujer embarazada $^{9,10}, 2$ casos de etiología biliar ${ }^{11,12}, 1$ caso de pancreatitis idiopática ${ }^{13}$, un caso de PA en embarazo sin asociación a hiperlipidemia ${ }^{14}$ y en 1 caso no se describe el origen de la pancreatitis ${ }^{8}$. En este trabajo, presentamos tres nuevos casos de AQPA. Se revisa la literatura y se analizan las características de los casos publicados y el tratamiento de esta complicación poco conocida por infrecuente.

\section{Pacientes y Métodos}

Se recogen los datos clínicos y evolutivos de los tres pacientes diagnosticados en nuestro Servicio de Cirugía de AQPA en los últimos 10 años. Se ha definido la AQPA como la presencia de linfa en cavidad abdominal de pacientes con episodio de pancreatitis aguda reciente y sin antecedente traumático, infeccioso, neoplásico o quirúrgico que pudiera ser el responsable de la misma. De otro lado, se ha solicitado al Servicio de Documentación, el número de pacientes ingresados en los últimos 10 años con diagnóstico de pancreatitis aguda.

Se recogen los datos epidemiológicos, causa de la pancreatitis, diagnóstico de la $\mathrm{AQ}$, procedimiento quirúrgico realizado, pérdidas de linfa, tratamiento y duración de la AQ, de todos los casos documentados, incluidos los nuestros.

\section{Resultados}

Sobre un total de 2.188 pacientes ingresados en nuestro hospital con diagnóstico de pancreatitis aguda en los últimos 10 años, se han diagnosticado tres casos de AQPA $(0,13 \%)$ :

\section{Caso 1}

Varón de 39 años; antecedentes de cólicos biliares y pancreatitis aguda grave (PAG) que precisó ingreso en la Unidad de Cuidados Intensivos (UCI) durante un mes, siendo dado de alta hospitalaria con diagnóstico de PAG de origen biliar con pseudoquiste residual de $16 \mathrm{~cm}$ y ascitis pancreática.

Reingresa a los 15 días con cuadro de dolor y distensión abdominal, cansancio y pérdida ponderal ( $2 \mathrm{~kg}$ en 15 días). Analítica de urgencias dentro de la normalidad. Se practicó una TC (Figura 1) en la que se informa de pseudoquiste de $16 \times 7 \times 13 \mathrm{~cm}$ con presencia de áreas hiperdensas en su interior que sugieren sangrado. Ascitis en espacio perihepático, periesplénico, ambas goteras paracólicas y en pelvis.

Se practica Angiografía con intención embolizante pero no se confirma sangrado activo. Dado que han pasado más de 6 semanas del episodio de PAG, se programa para colecistectomía y quistoyeyunostomía; en el acto operatorio se encuentran más de 3 litros de líquido de aspecto lechoso proveniente de retroperitoneo que salen a presión al realizar la laparotomía (Figura 2) y gran pseudoquiste con tejido necrótico en su interior. Se practicó Quistoyeyunostomia en Y de Roux y colecistectomía. Se realizaron lavados de cavidad y se dejó drenaje tipo Penrose. No se visualizó lesión linfática.

Bioquímica del líquido: Amilasa: $169 \mathrm{U} / \mathrm{l} ; \mathrm{pH}$ : 8; Proteínas: 4,3 mg/dl; glucosa: $96 \mathrm{mg} / \mathrm{dl}$, LHD: 275 U/l, Sodio: $136 \mathrm{mEq} / 1$; Potasio: $3,6 \mathrm{mEq} / \mathrm{l}$ y Triglicéridos: $537 \mathrm{mg} / \mathrm{dl}$. Cultivo: negativo.

El postoperatorio cursó con drenaje seroso que viró a quiloso una vez reiniciada la alimentación oral $\mathrm{al} 4^{\circ}$ día postoperatorio (DPO). Se instauró un tratamiento con octeótrido a dosis de $0,1 \mathrm{mg} / 8 \mathrm{~h}$, espironolactona, dieta sin grasas y suplementos de triglicéridos de cadena media (MTC), proteínas y vitaminas. La evolución del débito del drenaje quiloso a partir del $5^{\circ}$ DPO fue de 510, 490, 460, 400 cc, 200, 150, 10 (Figura 3), siendo retirado en el $12^{\circ}$ DPO y dado de alta en el día 13. 
Seguimiento en consulta externa: el paciente está asintomático con dieta normalizada y sin recurrencia al año.

\section{Caso 2}

Varón de 71 años que acude a urgencias con cuadro de dolor en hipocondrio derecho y sensación distérmica de $48 \mathrm{~h}$ de evolución. Antecedentes: Pancreatitis aguda de origen biliar hace un mes, diabetes mellitus tipo II, hipertensión arterial, hipercolestero-

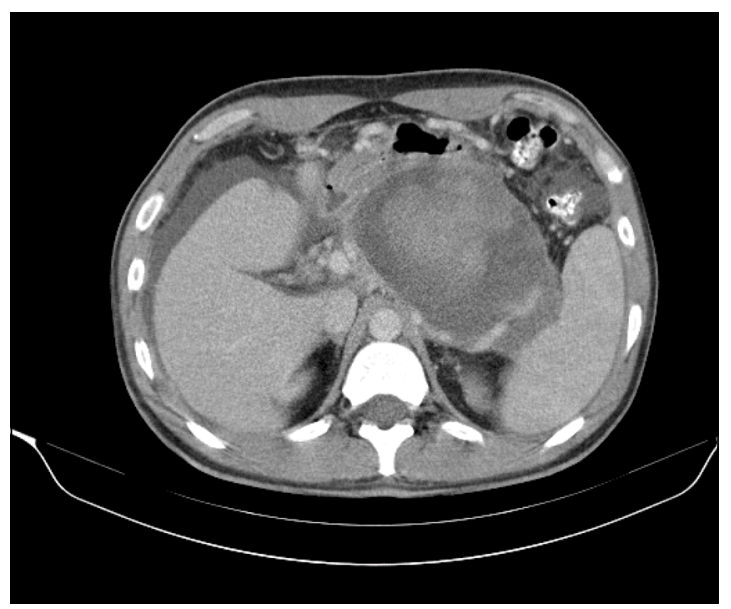

Figura 1. TC caso 1: Pseudoquiste de $16 \times 7 \times 13 \mathrm{~cm}$ con presencia de áreas hiperdensas en su interior que sugieren sangrado. Ascitis en espacio perihepático, periesplénico, ambas goteras paracólicas y en pelvis. lemia, estenosis mitral, bloqueo aurículo-ventricular de primer grado, accidente cerebrovascular con hemiparesia izquierda recuperada y neoplasia renal izquierda tratada mediante nefrectomía izquierda y radioterapia abdominal hace 6 años. Hipersensibilidad a contraste yodado intravenoso. En tratamiento con clopidogrel. Ingresa por cuadro compatible con colecistitis aguda; es intervenido de urgencia, encontrando colecistitis aguda gangrenosa con absceso perivesicular y realizándose colecistectomía, lavado

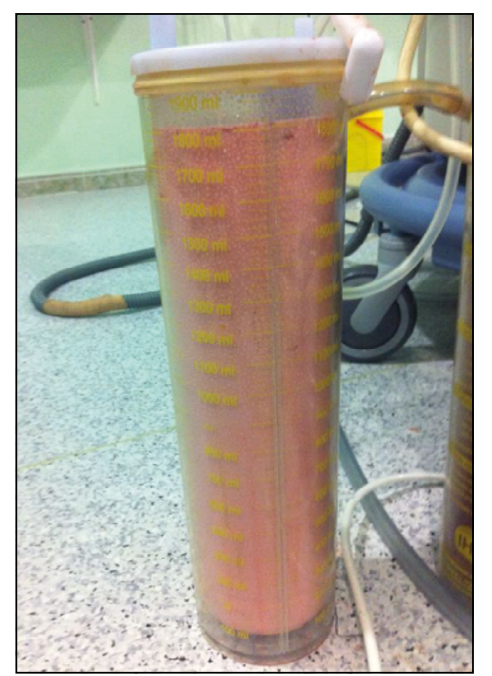

Figura 2. Colector del aspirador conteniendo material de aspecto lechoso, caso 1 .

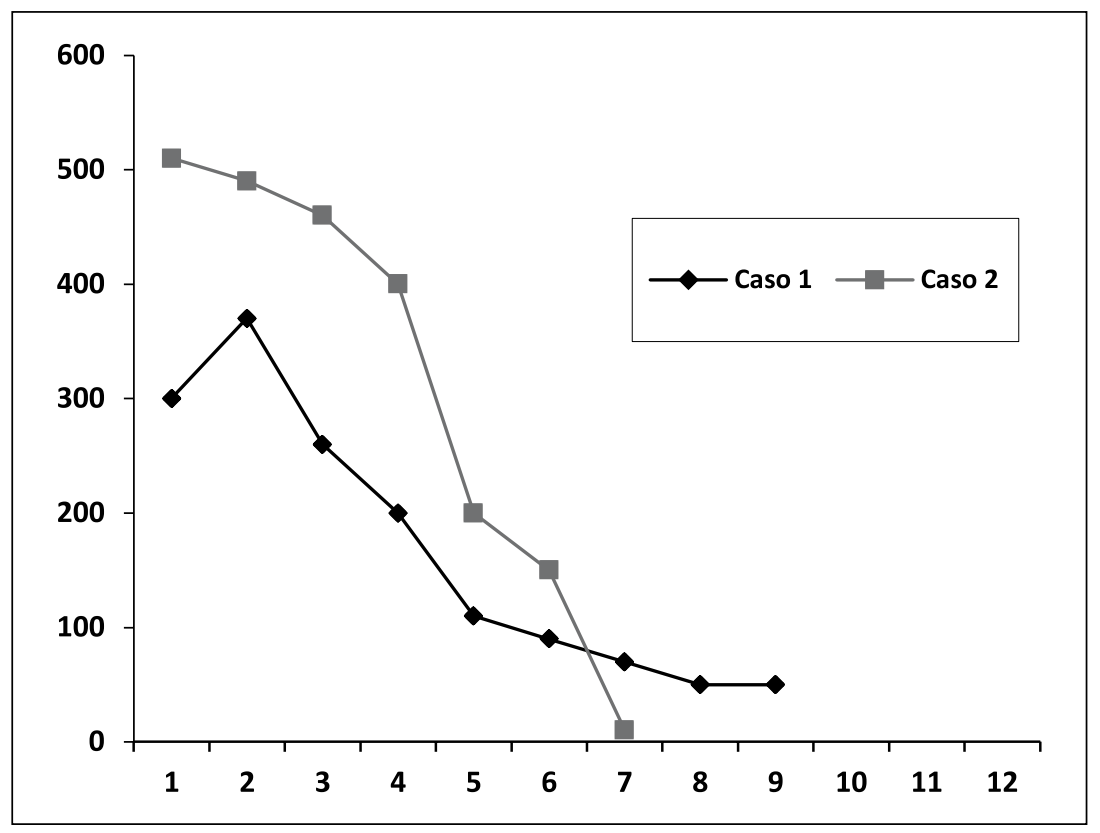

Figura 3. Evolución de la pérdida linfática en $\mathrm{cc}$, una vez instaurado el tratamiento, en los pacientes 1 y 2 . Eje $\mathrm{X}$ : días; Eje Y: Débito en cc. 
y drenaje de cavidad abdominal. Al $3^{\circ}$ DPO, $24 \mathrm{~h}$ tras iniciar dieta oral, el drenaje, inicialmente serohemático, presenta un aspecto lechoso (Figura 4) con débito de $300 \mathrm{cc} / 24 \mathrm{~h}$ que se confirmó como linfa tras el análisis bioquímico (glucosa $133 \mathrm{mg} / \mathrm{dl}$, colesterol $108 \mathrm{mg} / \mathrm{dl}$, triglicéridos $2.222 \mathrm{mg} / \mathrm{d}$ ). Cultivo: negativo. Se instauró un tratamiento con dieta hiperproteica, sin grasas, suplementos de MTC y octeótrido $0,1 \mathrm{mg} / 8 \mathrm{~h}$. El débito linfático fue de 300 , 370, 260, 200, 110, 90, 70, 50 y 50 cc (Figura 3). El paciente fue dado de alta hospitalaria con tratamiento dietético al $8^{\circ} \mathrm{DPO}$ y manteniendo el drenaje que fue retirado de forma ambulatoria a los 22 días de la intervención. Seguimiento de consulta: el paciente se encuentra asintomático, con dieta pobre en grasas $\mathrm{y} \sin$ recurrencia.

\section{Caso 3}

Mujer de 73 años de edad que ingresa en UCI por cuadro de PAG de etiología biliar que evoluciona a síndrome compartimental con una presión intraabdominal mantenida de $22 \mathrm{mmHg}$ y disfunción múltiple de órganos en las primeras $48 \mathrm{~h}$. Antecedentes: Isquemia cerebral transitoria hace un año, hipertensión arterial y obesidad. Al ingreso en UCI se realiza una TC que informa de colelitiasis, pancreatitis grado E de Balthazar y líquido libre peritoneal y retroperitoneal (Figura 5). La paciente es intervenida encontrando necrosis pancreática y extrapancreática así como $1.300 \mathrm{cc}$ de líquido de aspecto lechoso que en un principio se describió como ascitis pancreática pseudopurulenta y del que se tomaron muestras para cultivo, citología y bioquímica. Se realizó necrosectomía extensa, lavados de cavidad y se dejó una laparostomía protejida con bolsa tipo Bogotá. El cultivo y la citología fueron negativos. La bioquímica mostró amilasa: $7.000 \mathrm{U} / 1$ pero también triglicéridos de $2.500 \mathrm{mg} / \mathrm{dl}$.

Dado que estaba intubada, se indicó nutrición parenteral total (NTP) y octeótrido además de las medidas y tratamiento de UCI (drogas vasoactivas, diuréticos, antibióticos, oxigenoterapia, etc.). Tras una discreta mejoría en el postoperatorio inmediato, la evolución fue tórpida, no remontando en ningún momento y falleciendo finalmente a los 4 días de soporte intensivo.

Los casos de AQPA documentados hasta 2013, incluidos estos tres, se recogen en la Tabla 1 . Se corresponden con 15 pacientes, 7 hombres y $8 \mathrm{mu}$ jeres, con edades entre 24-78 años. Las causas más frecuentes de la pancreatitis fueron: alcohol $(33,3 \%)$, litiasis biliar (33,3\%) e hiperlipidemia en un 13,3\% ( 2 pacientes, una de ellas embarazada). El diagnóstico fue en todos los casos "de visu" intra o postoperatoriamente, y en un caso por drenaje a través de un catéter de diálisis peritoneal. Se encontraron cifras

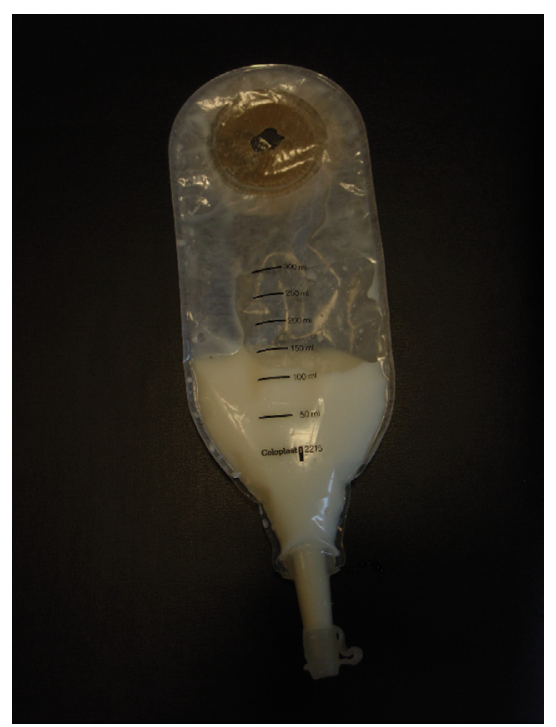

Figura 4. Aspecto del drenaje del paciente $\mathrm{N}^{\circ} 2$.

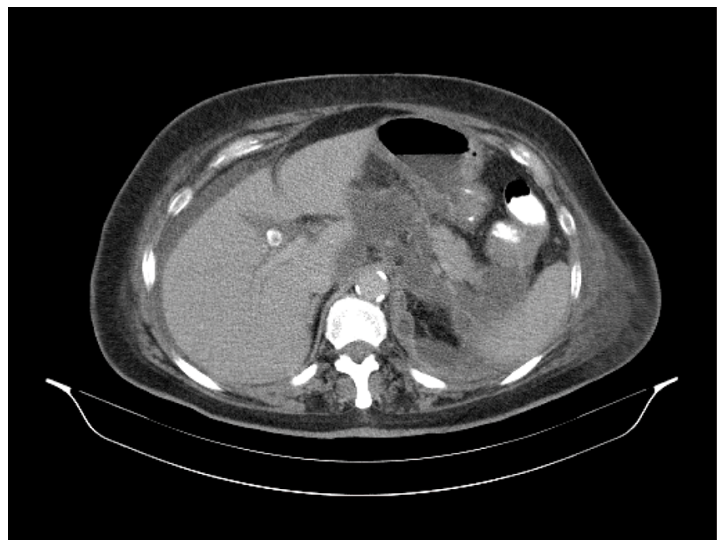

Figura 5. TC caso 3: Colelitiasis. Pancreatitis grado E de Balthazar y líquido libre peritoneal y retroperitoneal.

de triglicéridos en el líquido ascítico entre 134-8.030 $\mathrm{mg} / \mathrm{dl}$ con una media de $1.671,66 \mathrm{mg} / \mathrm{dl}$. Tratamiento de la AQ y duración de la misma: En 1 caso sólo con drenajes ( 7 días); dos casos con dieta pobre en grasas (60 y 21 días); dieta pobre en grasas y octeótrido: 3 pacientes (22,7 y 90 días); sólo NTP en 3 pacientes. NTP y Octeótrido: 4 pacientes $(5,7,15 \mathrm{y}$ 48 días). En un caso no se determina el tratamiento realizado para la $\mathrm{AQ}$ y en nuestra paciente número 3 se instauró NTP y octeótrido pero no es valorable dada la evolución fulminante de la pancreatitis con éxitus a los 4 días.

La resolución de la AQ si se realizó un tratamiento con octeótrido fue en 27,1 días, en tanto que con tratamientos sin este (con independencia del régimen dietético) fue en 46,5 días. 
Tabla 1. Casos documentados de ascitis quilosa asociados a pancreatitis aguda. Se incluyen nuestros tres pacientes al final de la serie

\begin{tabular}{|c|c|c|c|c|c|c|c|c|}
\hline Autor, Año & Sexo & Edad & Causa PA & Diagnóstico & $\begin{array}{c}\text { Débito } \\
\text { inicial en cc }\end{array}$ & $\begin{array}{l}\text { Triglicéridos } \\
\text { Mgr/dl }\end{array}$ & Tratamiento & $\begin{array}{l}\text { Dura- } \\
\text { ción }\end{array}$ \\
\hline Golfarb, 1984 & $\mathrm{H}$ & 43 & Alcohol & $\begin{array}{l}\text { Toracentesis/ } \\
\text { Paracentesis }\end{array}$ & 475 & 8.034 & Drenajes & $7 d$ \\
\hline $\begin{array}{l}\text { Pérez-Fontan, } \\
1993\end{array}$ & M & 66 & Biliar & $\begin{array}{l}\text { Drenaje catéter } \\
\text { Diálisis peritoneal }\end{array}$ & 1.800 & 1.515 & NTP & $\mathrm{NC}$ \\
\hline $\begin{array}{l}\text { Ben-Ami, } \\
1999\end{array}$ & M & 68 & Alcohol/biliar & Intraoperatorio & 2.500 & 398 & DSG/MTC & $60 \mathrm{~d}$ \\
\hline Liu, 2001 & M & 24 & Embarazo & Intraoperatorio & $\mathrm{NC}$ & $\mathrm{NC}$ & NTP & $60 \mathrm{~d}$ \\
\hline Chuang, 2006 & M & 28 & $\begin{array}{l}\text { Embarazo/ } \\
\text { Hiperlipidemia }\end{array}$ & Intraoperatorio & 2.500 & 577 & NTP/DSG/MTC & $45 \mathrm{~d}$ \\
\hline Kahn, 2007 & $\mathrm{H}$ & 54 & Hiperlipidemia & Paracentesis & $\mathrm{NC}$ & 3.200 & DSG & $21 d$ \\
\hline $\begin{array}{l}\text { Al-Ghamdi, } \\
2007\end{array}$ & $\mathrm{H}$ & 50 & Alcohol & Paracentesis & 5.000 & 1.400 & $\begin{array}{l}\text { NTP } \\
\text { Octeótrido }\end{array}$ & $48 \mathrm{~d}$ \\
\hline Smith, 2009 & $\mathrm{H}$ & 46 & Alcohol & Intraoperatorio & $\mathrm{NC}$ & 2.280 & $\mathrm{NC}$ & $4 d$ \\
\hline $\begin{array}{l}\text { Gómez } \\
\text { Martin, } 2012\end{array}$ & M & 68 & Idiopática & $\begin{array}{l}\text { Drenaje } \\
\text { radioguiado }\end{array}$ & $\mathrm{NC}$ & 4.000 & $\begin{array}{l}\text { NE/DSG/MTC } \\
\text { Octeótrido }\end{array}$ & $90 \mathrm{~d}$ \\
\hline $\begin{array}{l}\text { Georgios, } \\
2012\end{array}$ & $\mathrm{H}$ & 46 & Alcohol & Intraoperatorio & $\mathrm{NC}$ & 2.800 & $\begin{array}{l}\text { NTP/DSG/MTC } \\
\text { Octeótrido }\end{array}$ & $15 \mathrm{~d}$ \\
\hline Zárate, 2013 & M & 55 & Alcohol & $\begin{array}{l}\text { Drenaje } \\
\text { radioguiado }\end{array}$ & 700 & 200 & $\begin{array}{l}\text { NTP } \\
\text { Octeótrido }\end{array}$ & $7 \mathrm{~d}$ \\
\hline Zárate, 2013 & M & 78 & $\mathrm{Nc}$ & Postoperatorio & 750 & 134 & $\begin{array}{l}\text { NTP } \\
\text { Octeótrido }\end{array}$ & $5 \mathrm{~d}$ \\
\hline Caso 1 & $\mathrm{H}$ & 39 & Biliar & Intraoperatorio & 3.000 & 537 & $\begin{array}{l}\text { DSG/MTC } \\
\text { Octeótrido }\end{array}$ & $7 \mathrm{~d}$ \\
\hline Caso 2 & $\mathrm{H}$ & 71 & Biliar & Postoperatorio & 300 & 2.222 & $\begin{array}{l}\text { DSG/MTC } \\
\text { Octeótrido }\end{array}$ & $18 \mathrm{~d}$ \\
\hline Caso 3 & M & 73 & Biliar & Intraoperatorio & 1.300 & 2.500 & NV & NV \\
\hline
\end{tabular}

H: hombre; M: mujer; NTP: nutrición parenteral; DSG: dieta sin grasas; MTC: triglicéridos de cadena media; NE: nutrición enteral; NC: no consta; NV: no valorable

\section{Discusión}

La ascitis quilosa (AQ) consiste en la presencia de linfa libre en la cavidad abdominal, sea de origen torácico o intestinal. Para definirla se han de cumplir una serie de criterios entre los que destacan la presencia de líquido de aspecto lechoso, con cultivo negativo y, sobre todo, con la presencia de más de 120 o $200 \mathrm{mg} / \mathrm{dl}$ de triglicéridos, según autores ${ }^{2,3,8,15}$. La causa más frecuente de AQ en el adulto, es el traumatismo directo de los linfáticos y dentro de las causas no traumáticas, las neoplasias y la cirrosis ocupan los primeros lugares ${ }^{1,2}$. Los casos asociados a pancreatitis aguda (AQPA) son escasos, y se reducen a publicaciones de casos clínicos aislados ${ }^{4-14}$. Fisiopatológicamente la AQPA podría producirse por una obstrucción linfática secundaria a la inflamación pancreática y peripancreática o bien por lesión directa causada por la suelta de enzimas pancreáticas ${ }^{2,12-15}$. No se puede precisar cuál es la incidencia real de AQPA, sobre todo teniendo en cuenta que, de un lado, la PA con mucha frecuencia se acompaña de líquido libre peritoneal que no suele analizarse y que en la TC presenta, al igual que el quilo, densidad de agua $^{16,17}$; de otro lado, la ascitis quilosa leve puede autolimitarse y no dar ninguna sintomatología al igual que se acaba reabsorbiendo el líquido libre de una pancreatitis aguda. Esto hace que todos los casos se diagnostiquen una vez drenada la AQ. En nuestra serie, la incidencia ha sido de 1,3 casos/100 PA, aunque en el caso 2 pueda haber influido de algún modo la radioterapia. Si descartamos este caso, 
la incidencia de AQPA sería de 0,09 casos por cada $100 \mathrm{PA}$, pero es posible que esté infradiagnosticada; de hecho, Zárate en un período de 2 años y medio, encuentra 4 casos de fístula quilosa de los cuales dos van asociados a episodio de pancreatitis aguda ${ }^{8}$. Tal vez si se realizaran paracentesis en todos los casos de pancreatitis aguda con líquido libre se encontrarían más casos de $\mathrm{AQ}$.

La AQPA generalmente debuta con aumento progresivo del perímetro abdominal, dolor por distensión y sensación de cansancio días o semanas después del episodio de PA, (como ocurrió con nuestro primer paciente); en muy pocos casos comienza a la vez que la PA emulando un cuadro de peritonitis que lleva al paciente al quirófano ${ }^{6,7,9}$.

El diagnóstico de AQ requiere un alto índice de sospecha y en los casos de AQPA más, tal y como se ha explicado anteriormente; todos los casos de AQPA revisados, incluidos los nuestros, se han diagnosticado "de visu" ya sea mediante paracentesis, drenaje radioguiado, laparoscopia o laparotomía y ninguno ha sido sospechado por la imagen de la TC (Tabla 1).

Los objetivos del tratamiento son: 1) el mantenimiento o mejora de la nutrición; 2) la disminución de la tasa de formación de quilo y 3) la corrección del trastorno subyacente ${ }^{3}$ : Se puede iniciar con una dieta sin grasas (DSG) con suplementos proteicos, vitamínicos y triglicéridos de cadena media (MTC) que se absorben sin necesidad de síntesis de quilomicrones y pasan directamente a la circulación portal. La nutrición parenteral total, se encontraría en un siguiente escalón terapéutico dentro del tratamiento conservador. Con NTP se consigue bajar el flujo linfático de $220 \mathrm{cc} / \mathrm{kg} / \mathrm{h}$ a $1 \mathrm{cc} / \mathrm{kg} / \mathrm{h}^{2,18}$, consiguiendo la resolución entre 14 y 45 días ${ }^{19}$. Tanto la somatostatina como su análogo, el octreótido, se han usado en el tratamiento de la AQ y parece que, además de la presión portal y el flujo esplácnico, disminuyen el flujo linfático, así como su concentración en triglicéridos $^{19-22}$; el octeótrido es más fácil de administrar por lo que se ha impuesto frente a la somatostatina. Nuestros pacientes 1 y 2 fueron tratados con DSG y suplementos más octeótrido a dosis de $0,1 \mathrm{mg} / 8 \mathrm{~h}$ consiguiendo la resolución completa en 7 y 18 días respectivamente. Todos los casos de AQPA revisados $^{4-14}$ se resolvieron con tratamiento conservador (Tabla 1). Cuando se ha añadido el octeótrido sea a NTP o a DSG, la resolución completa se produce a los 27,1 días de media, en tanto que los pacientes tratados sin octeótrido, se resuelven en una media de 46,5 días, lo que hace pensar que efectivamente el análogo de la somatostatina es eficaz frente a la $\mathrm{AQ}$ y debe tenerse como tratamiento de primera línea como ya indican otros autores ${ }^{8,19-22}$.

Cuando la AQ no es controlable se puede realizar
Tabla 2. Clasificación de ascitis quilosa propuesta por Van der Gaag

\begin{tabular}{|lccc|}
\hline & Grado A & Grado B & Grado C \\
E. general & Bueno & Mediano & Regular \\
Infección & $\mathrm{NO}$ & $\mathrm{NO}$ & $\mathrm{SI}$ \\
AQ Evidente en ECO/TC & - & - & + \\
Duración & $<7 \mathrm{~d}$ & $7-14 \mathrm{~d}$ & $>14 \mathrm{~d}$ \\
Tt $^{\circ}$ dietético & $\mathrm{Si} / \mathrm{No}$ & $\mathrm{DSG}$ & $\mathrm{NTP}$ \\
Drenaje persistente & $\mathrm{No}$ & $\mathrm{Si}$ & $\mathrm{Si}$ \\
Cirugía* & $\mathrm{No}$ & $\mathrm{No}$ & $\mathrm{Si}$ \\
Larga estancia* $^{*}$ & $\mathrm{No}$ & $\mathrm{Si}$ & $\mathrm{Si}$ \\
Reingreso* $^{*}$ & $\mathrm{No}$ & $\mathrm{No}$ & $\mathrm{Si} / \mathrm{No}$ \\
\hline
\end{tabular}

*A causa de la ascitis quilosa. DSG: dieta sin grasas; NTP: Nutrición parenteral.

una linfogammagrafía que es el gold estándar para reconocer una lesión linfática, e intentar embolización radioguiada; si esta no es efectiva, habrá que recurrir a la cirugía con técnicas que van desde ligaduras de los linfáticos a shunt peritoneovenoso ${ }^{2,19}$.

Dependiendo de la gravedad del cuadro de AQ se utilizará uno u otro tratamiento. Van der $\mathrm{Gaag}^{23}$ ha propuesto una clasificación de la ascitis quilosa post duodenopancreatectomía, que la divide en tres grados (A, B y C) atendiendo al estado general del paciente, infección de la ascitis, duración, AQ evidente en ECO o TC, tratamiento requerido, estancia hospitalaria y necesidad de reingreso (Tabla 2). Según esta clasificación casi todos los casos descritos corresponderían a un grado B.

En general podemos aplicar la pauta siguiente:

- Leves-moderadas (A-B de Van der Gaag): Dieta sin grasas y suplementos de proteínas y vitaminas, además de octeótrido durante 3-6 semanas.

- Graves (C) o No mejoría: NTP y octeótrido (la dosis se puede aumentar hasta $0,4 \mathrm{mg} / 8 \mathrm{~h}$ ).

- No mejoría y más de $1.500 \mathrm{cc} / \mathrm{d}$ durante $1 \mathrm{se}-$ mana: Linfogammagrafía e intervención según hallazgos.

\section{Conclusiones}

1. La Ascitis quilosa secundaria a pancreatitis aguda (AQPA) es un cuadro infrecuente que puede presentarse de forma aguda o subaguda, unas semanas alrededor del episodio de pancreatitis.

2. Se diagnostica "de visu" ya que por TC es imposible diferenciar de los hallazgos de la propia pancreatitis. 
3. El tratamiento con NTP o DSG y suplementos debe asociarse de entrada a octeótrido a la vista de los resultados publicados.

4. Ninguna AQPA de las mencionadas ha precisado tratamiento quirúrgico.

\section{Referencias}

1. Steinemann DC, Dindo D, Clavien PA, Nocito A. Atraumatic Chylous Ascites: Systematic Review on Symptoms and Causes. J Am Coll Surg. 2011;212:899905.

2. Aalami OO, Allen DB, Organ CH. Chylous ascites a collective review. Surgery 2000;128,761-8.

3. Cardenas A, Chopra S. Chylous ascites. Am J Gastroenterol. 2002;97:1896-900.

4. Goldfarb JP. Chylous effusions secondary to pancreatitis: case report and review of the literature. Am J Gastroenterol. 1984;79:133-5.

5. Al-Ghamdi MY, Bedi A, Reddy SB, Tanton RT, Peltekian KM. Chylous ascites secondary to pancreatitis: management of an uncommon entity using parenteral nutrition and octeotride. Dig Dis Sci. 2007;52:2261-4.

6. Smith EK, EK E, Croagh D, Spain LA, Farrell S. Acute chylous ascites mimicking acute apendicitis in a patient with pancreatitis. World J Gastroenterol. 2009;15:484952.

7. Georgiou GK, Harissis H, Mitsis M, Batsis H, Fatouros M. Acute chylous peritonitis due to acute pancreatitis. World J Gastroenterol. 2012;18:1987-90.

8. Zárate Moreno F, Oms Bernad LM, Mato Ruiz R, Balaguer del Ojo C, Sala Pedros J, Campillo Alonso F. Eficacia del octeótrido en el tratamiento de la fístula quilosa asociada a enfermedades pancreáticas. Cir Esp. 2013;91:237-42.

9. Chuang SC, Lee KT, Wang SN, Kuo KK, Chen JS. Hypertriglyceridemia-associated Acute Pancreatitis with Chylous Ascites in Pregnancy. J Formos Med Assoc. 2006;105:583-7.

10. Khan FY, Matar I. Chylous ascites secondary to hyperlipidemic pancreatitis with normal serum amilase and lipase. World J Gastroenterol. 2007;13:480-2.

11. Pérez Fontán M, Pombo F, Soto A, Pérez Fontán FJ,
Rodríguez-Carmona A. Chylous ascites associated with acute pancreatitis in a patient undergoing continuous ambulatory peritoneal dialysis. Nephron. 1993;63:45861.

12. Ben-Ami H, Nagachandran P, Assalia A, Edoute Y. Acute transient chylous ascites associated with acute biliary pancreatitis. Am J Med Sci. 1999;318:122-3.

13. Gómez Martin JM, Martínez-Molina E, Sanjuanbenito A, Martin-Illana E, Arrieta F, Balsa JA, et al. Chylous ascites secondary to acute pancreatitis: a case report and review of literature Nutr Hosp. 2012;27:314-8.

14. Liu CJ, Yen CL, Chang JJ, Lee TS, Fan KM. Chylous ascites in acute pancreatitis during pregnancy: case report. Chang Gung Med J. 2001;24:324-8.

15. Jiménez CE. Ascitis quilosa: Presentación de un caso y revisión de la fisiopatología. Rev Colomb Cir. 2004;19:76-84.

16. Patten RM, Calkins CM, Moore EE. Isolated traumatic rupture of the cisterna chyli: CT diagnosis. J Comput Assist Tomogr. 1999;23:701.

17. Wachsberg RH, Cho KC. Chyloperitoneum: CT diagnosis. Clin Imaging 1994;18:273.

18. García G, Aguilar C, Murcia R, Espinosa P. Manejo conservador de fístula linfática postquirúrgica. Cir Cir. 2005;73:307-10.

19. Leibovitch I, Mor Y, Golomb J, Ramon J. The diagnosis and management of postoperative chylous ascites. J Urol. 2002;167:449-57.

20. Neveu R, Fernández R, Bucholtz M, González M, Rodríguez J, Trujillo C, y cols. Manejo conservador de la fistula quilosa con somatostatina. Rev Chil Cir. 2006;58:219-23.

21. Qi Huang, Zhi-Wei Jiang, Ning Li, Jie-Shou Li. Chylous ascites: Treated with total parenteral nutricion and somatostatin. World J Gastroenterol. 2004;10:258891.

22. Shapiro AM, Bain VG, Sigalet DL, Kneteman NM. Rapid resolution of chylous ascites after liver transplantation using somatostatin analog and total parenteral nutrition. Transplantation 1996;61:1410-11.

23. Van der Gaag NA, Verhaar AC, Haverkort EB, Busch ORC, van Gulik TM, Gouma DJ. Chylous ascites after pancreatoduodenectomy: Introduction of a grading system. J Am Coll Surg. 2008;207:751-7. 